\title{
Nitrogen Sulfide (NS) In Star Forming Regions
}

\author{
Douglas McGonagle William Irvine \\ Young Minh * \\ Five College Radio Astronomy Observatory \\ University of Massachusetts at Amherst, USA
}

Gas phase models of ion molecule chemistry have been rather successful in matching the observed abundances of small interstellar molecules containing carbon, hydrogen, and oxygen. However, the situation is somewhat less clear for nitrogen-containing species, partly because the important initiating reaction $\mathrm{N}^{+}+\mathrm{H}_{2}$ is slightly endothermic; and for sulfurcontaining molecules, where it remains uncertain whether it is necessary to invoke surface reactions on grains to match the observed abundances. As a relatively simple species, the abundance of nitrogen sulfide should provide a good test of the models of the coupled chemistry of nitrogen and sulfur. Until very recently only two molecules containing both these elements were known in the interstellar medium, NS and HNCS, and both have been observed only in Sgr B2. We have therefore undertaken a survey for interstellar NS in Galactic molecular clouds using the FCRAO 14 -meter telescope. The ${ }^{2} \Pi_{1 / 2}, J=5 / 2 \rightarrow 3 / 2$, transition has in fact been detected in many regions of massive star formation (see table).

The three dominant hyperfine components of the lower frequency $\Lambda$-doublet component were detected in almost all cases. The ratios of the component intensities differ from the predicted intrinsic relative intensity ratios, thus suggesting that in several sources the emission is not optically thin, which in turn implies that the NS source size is small compared to the beam size (45"). The beam average column densities for NS derived from these observations are $N \sim 10^{13}-10^{14} \mathrm{~cm}^{-2}$. Using a canonical molecular hydrogen column density of $10^{23} \mathrm{~cm}^{-2}$ we estimate the fractional abundance to be $f \sim 10^{-10}$ relative to $\mathrm{H}_{2}$, e.g. in Orion-KL. These abundances are $2-3$ orders of magnitude larger than the predictions of the gas phase models for an Orion type cloud by Miller and Herbst (A\&A, 1990, 231, 466).

\footnotetext{
* Now at DRAO, South Korea.
} 


\section{Nitrogen Sulfide in Star Forming Regions}

\begin{tabular}{|c|c|c|c|c|c|c|c|c|c|c|}
\hline \multirow[t]{2}{*}{ Source } & \multicolumn{3}{|c|}{$\alpha(1950)$} & \multicolumn{3}{|c|}{$\delta(1950)$} & \multirow{2}{*}{$\begin{array}{c}V_{l s r} \\
\left(k m s^{-1}\right)\end{array}$} & \multirow{2}{*}{$\begin{array}{l}T a^{*} \\
(\mathrm{~K})\end{array}$} & \multirow{2}{*}{$\begin{array}{c}\mathrm{I} \\
\left(K k m s^{-1}\right)\end{array}$} & \multirow{2}{*}{$\begin{array}{c}N_{N S} \\
\left(\mathrm{~cm}^{-2}\right)\end{array}$} \\
\hline & hh & $\mathrm{mm}$ & ss.s & dd & $\mathrm{mm}$ & ss.s & & & & \\
\hline DR21(OH) & 20 & 37 & 15.0 & 42 & 12 & 8.0 & -2.9 & 0.08 & $1.06 \pm 0.08$ & $3 \times 10^{13}$ \\
\hline $\mathrm{G} 34.3+0.2$ & 18 & 50 & 46.0 & 1 & 11 & 10 & +57.8 & 0.17 & $2.04 \pm 0.16$ & $6 \times 10^{13}$ \\
\hline NGC7538(E) & 23 & 11 & 53.0 & 61 & 10 & 57.9 & -58.4 & 0.05 & $0.34 \pm 0.08$ & $1 \times 10^{13}$ \\
\hline NGC7538(N) & 23 & 11 & 36.6 & 61 & 11 & 48.0 & -66.7 & 0.05 & $0.61 \pm 0.13$ & $2 \times 10^{13}$ \\
\hline NGC7538(S) & 23 & 11 & 36.6 & 61 & 10 & 48.0 & -55.8 & 0.09 & $0.92 \pm 0.08$ & $3 \times 10^{13}$ \\
\hline ORION(S) & 5 & 32 & 44.8 & -5 & 25 & 60.0 & +6.4 & 0.07 & $0.74 \pm 0.21$ & $2 \times 10^{13}$ \\
\hline ORION(KL) & 5 & 32 & 47.0 & -5 & 24 & 23.0 & +7.6 & 0.14 & $1.96 \pm 0.23$ & $6 \times 10^{13}$ \\
\hline S140 & 22 & 17 & 41.2 & 63 & 3 & 41.0 & -8.0 & 0.05 & $0.38 \pm 0.08$ & $1 \times 10^{13}$ \\
\hline SGR(B2M) & 17 & 44 & 10.6 & -28 & 22 & 5.0 & +60.6 & 0.17 & $4.19 \pm 0.52$ & $1 \times 10^{14}$ \\
\hline W3(OH) & 2 & 23 & 17.3 & 61 & 38 & 57.9 & -46.7 & 0.10 & $0.82 \pm 0.10$ & $2 \times 10^{13}$ \\
\hline W51(MS) & 19 & 21 & 26.4 & 14 & 24 & 42.0 & +55.6 & 0.19 & $2.64 \pm 0.26$ & $8 \times 10^{13}$ \\
\hline W51(N) & 19 & 21 & 22.0 & 14 & 25 & 20.0 & +59.8 & 0.09 & $1.66 \pm 0.26$ & $5 \times 10^{13}$ \\
\hline
\end{tabular}

\section{NOTES:}

1) The integrated intensity, $I$, is over the three major hyperfine components of the ${ }^{2} \Pi_{1 / 2}$, $J=5 / 2 \rightarrow 3 / 2$ transition. Not corrected for beam efficiency $\left(\eta_{b}=0.55\right)$.

2) The total column density, $N_{N S}$, is corrected for beam efficiency and the unobserved portion of the lambda doubling and hyperfine structure. For all sources, $T_{\text {rot }}=30 \mathrm{~K}$ is assumed. 


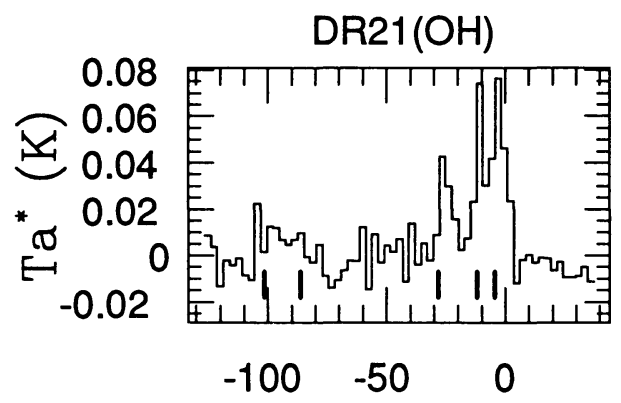

G34.3+0.2
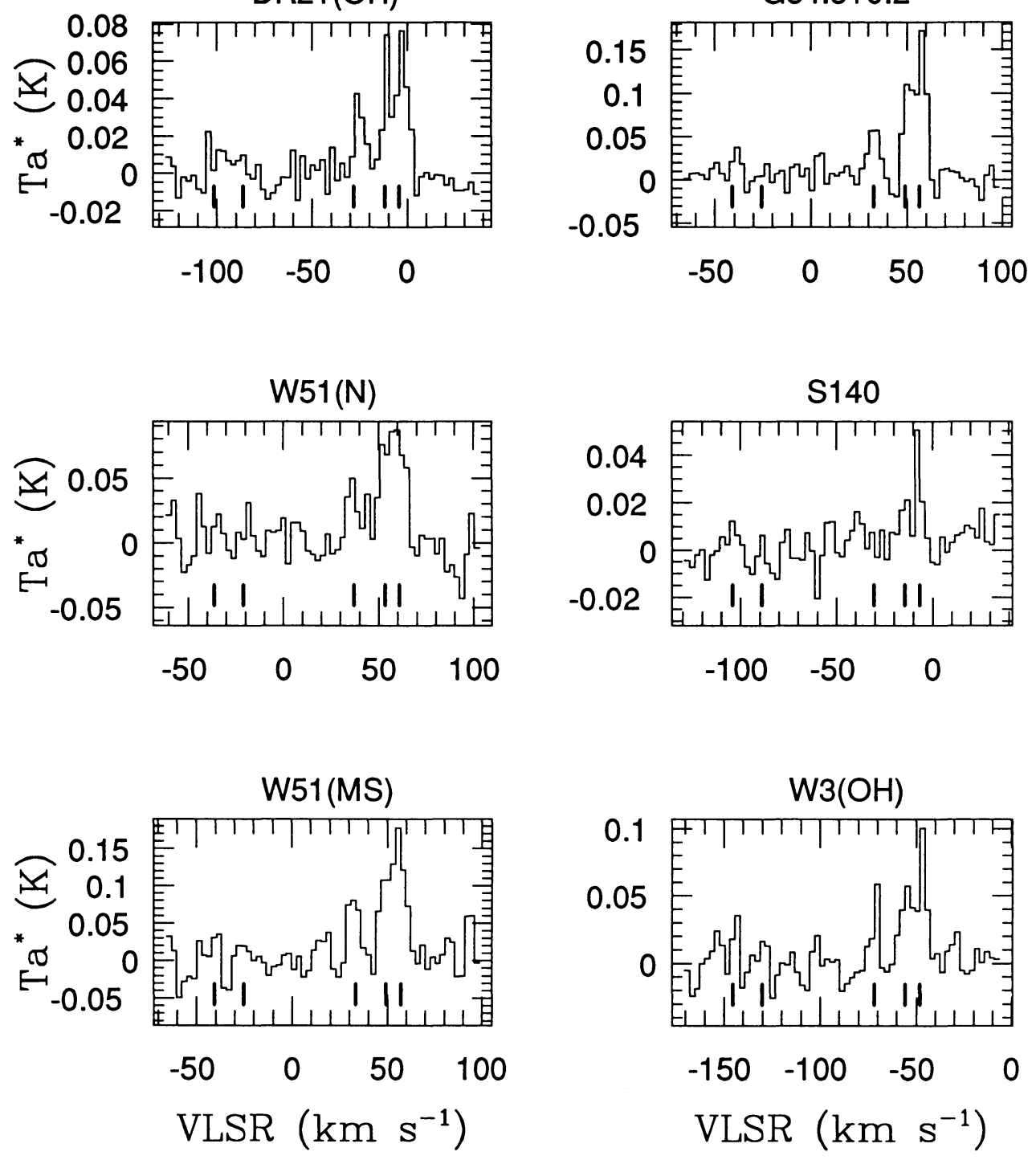

Figure 1: Spectra of the ${ }^{2} \Pi_{1 / 2}, J=5 / 2 \rightarrow 3 / 2$ transition of NS towards the sources listed in table 1. The spectral resolution is $1 \mathrm{MHz}$, and the spectral band includes the transitions three major hyperfine components. 

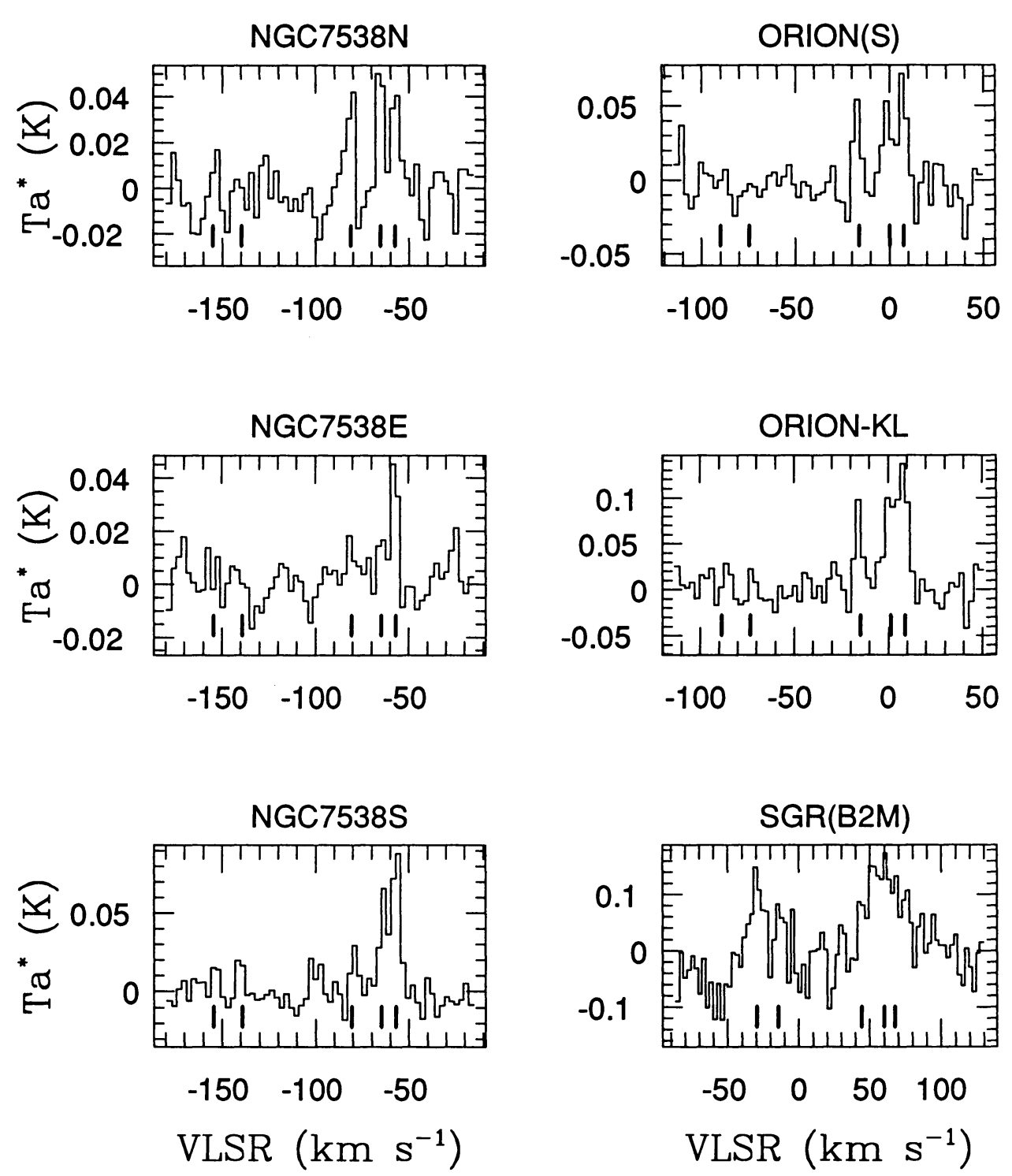

Figure 1: (cont) Spectra of the ${ }^{2} \Pi_{1 / 2}, J=5 / 2 \rightarrow 3 / 2$ transition of NS towards the sources listed in table 1 . The spectral resolution is $1 \mathrm{MHz}$, and the spectral band includes the three major hyperfine components. 